\title{
Network Dynamics Mediating Ipsilateral Motor Cortex Activity during Unimanual Actions
}

\author{
Timothy Verstynen ${ }^{1}$ and Richard B. Ivry ${ }^{2}$
}

\begin{abstract}
Executing difficult actions with the left hand results in bilateral activity of motor areas along the precentral gyrus. Using TMS and fMRI, we explored the functional relationship between primary (M1) and premotor areas during unimanual actions, focusing on M1 activity in the ipsilateral hemisphere. Single-pulse TMS revealed that the amplitude of motor-evoked potentials (MEPs), elicited in the stationary right-hand muscles following left M1 stimulation, fluctuated with the state of homologous muscles in the moving left hand. This ipsilateral excitability was pronounced when the left-hand movements were more
\end{abstract}

\section{INTRODUCTION}

The contralateral control of distal muscles is a fundamental characteristic of the primate motor system (Brinkman \& Kuypers, 1973). Nonetheless, neurophysiological studies in monkeys (Cisek, Crammond, \& Kalaska, 2003; Donchin, Gribova, Steinberg, Bergman, \& Vaadia, 1998; Tanji, Kazuhiko, \& Kazuko, 1988) and neuroimaging studies in humans (Hanakawa, Parikh, Bruno, \& Hallett, 2005; Verstynen, Diedrichsen, Albert, Aparicio, \& Ivry, 2005; Kobayashi, Hutchinson, \& Schlaug, 2003; Cramer, Finklestein, Schaechter, Bush, \& Rosen, 1999; Singh et al., 1998; Kawashima, Inoue, Sato, \& Fukuda, 1997; Kim et al., 1993; Shibasaki et al., 1993) have consistently reported activity in ipsilateral motor cortex during unimanual movements. This activation is weaker than that observed during contralateral limb movements, but is particularly strong during left-hand actions, regardless of an individual's handedness (Hanakawa et al., 2005; Verstynen et al., 2005; Kim et al., 1993).

This ipsilateral activity initially was thought to be centered in primary motor cortex, M1 (Kobayashi et al., 2003; Singh et al., 1998; Kawashima et al., 1997; Kim et al., 1993; Rao et al., 1993; Shibasaki et al., 1993), a conclusion consistent with transcranial magnetic stimulation (TMS) studies showing increased excitability of M1 during isometric contractions of ipsilateral hand muscles (Stinear, Walker, \& Byblow, 2001; Muellbacher, Facchini, Boroojerdi, \& Hallett, 2000; Stedman, Davey, \& Ellaway, 1998). However, recent imaging evidence is at odds with an M1 focus of ipsilateral activity. Across a number of studies, the location of

${ }^{1}$ University of Pittsburgh, ${ }^{2}$ University of California, Berkeley complex. We used fMRI to visualize the cortical dynamics during unimanual actions. Trial-by-trial fluctuations in ipsilateral M1 activity were correlated with contralateral M1 responses and this correlation increased with movement complexity. Consistent with previous studies, the left caudal precentral premotor area (pcPM) was engaged during movements of either hand. Following low-frequency rTMS over left pcPM, the correlation between the activity level in the two M1s increased. This finding indicates that left pcPM may regulate the unintentional mirroring of motor commands in M1 during unilateral movement. ipsilateral activity is shifted by approximately $2 \mathrm{~cm}$ in a lateral-anterior-ventral direction from the hand area of M1 (Hanakawa et al., 2005; Verstynen et al., 2005; Cramer et al., 1999). The center of this activation is on the medial bank of the precentral gyrus, an area corresponding to caudal aspects of dorsal premotor cortex (Hanakawa et al., 2005) and approximately coinciding with Brodmann's area 6a⿱ $($ Geyer, Matelli, Luppino, \& Zilles, 2000). We will refer to this region within premotor cortex on the precentral gyrus as pcPM.

Functionally, the fact that ipsilateral activity is in premotor cortex, rather than M1, suggests a role in planning and/or high-level control processes rather than processes primarily associated with motor execution. This hypothesis is supported by the observation that the magnitude of activity in ipsilateral motor areas increases with movement complexity (Verstynen et al., 2005; Rao et al., 1993; Shibasaki et al., 1993). Moreover, activity in pcPM is negatively correlated with the proficiency of the ipsilateral hand during these complex actions (Verstynen et al., 2005).

Defining what makes an action "complex" is problematic. Why is repeating a sequence of finger movements more complex than repeating the same movement with a single effector? Control theory in engineering provides one perspective for answering this question for hand movements (Ingram, Körding, Howard, \& Wolpert, 2008; Todorov, 2004; Weiss \& Flanders, 2004; Santello \& Soechting, 2000; Santello, Flanders, \& Soechting, 1998). Simultaneously flexing and extending all of the fingers (e.g., waving goodbye) involves a single degree of freedom centered about the metacarpophalangeal joint, leading to high covariance 
across the fingers. In contrast, sequential finger movements require different degrees of flexion, extension, abduction, and adduction at each point in time. In this way, each finger acts with relative independence, reducing covariance and increasing the overall degrees of freedom required to execute the movement. When considered in these terms, actions that engage ipsilateral motor areas and produce unintended mirror movements are those that involve relatively large degrees of freedom (e.g., Verstynen et al., 2005).

Although ipsilateral activity is centered in pcPM, there is also ample evidence to suggest that ipsilateral primary motor cortex itself is modulated during unimanual actions. TMS-induced motor-evoked potentials (MEPs) elicited in a resting hand via stimulation of M1 are elevated during isometric contractions produced by the other hand (Stinear et al., 2001; Muellbacher et al., 2000; Stedman et al., 1998). This suggests that the excitability of M1 itself changes during ipsilateral actions. This modulation of M1 can sometimes lead to overt and unintended movements in the "stationary" hand, or what are referred to as mirror movements. Mirror movements, although generally absent after childhood, may be manifest even in healthy adults when executing particularly complex and difficult actions with the other hand (Mayston, Harrison, \& Stephens, 1999).

Here we report a set of experiments that combine TMS and fMRI to explore the functional role of ipsilateral motor areas during unimanual movements. First, we tested the hypothesis that changes in M1 excitability during ipsilateral movements reflect a mirroring of the motor commands being controlled by contralateral motor cortex. Single-pulse TMS was used to test the prediction that variation in the amplitude of MEPs elicited in stationary, right-hand muscles will vary as a function of both the state of muscles in the moving left hand and of the complexity of the movement being executed. Second, we used fMRI to examine whether the BOLD signal in left M1 correlates with trial-by-trial changes in right M1 (see also Biswal, Yetkin, Haughton, \& Hyde, 1995). Specifically, we tested the hypothesis that increasing the complexity of a unimanual action will increase the degree to which a mirrored motor signal is expressed in ipsilateral M1. We predicted that the degree of coupling between left and right M1 would be stronger for complex actions compared to simple actions. Third, we used a combined TMS and fMRI procedure to test the hypothesis that pcPM mediates the degree of M1 coupling. Using repetitive TMS (rTMS), we disrupted activity in pcPM and tested whether this modulates the degree of coupling between left and right M1.

In short, the goals of this investigation were to use single-pulse TMS to probe the excitability of M1 directly, use fMRI to look at the cortical dynamics of precentral motor areas, and use a novel integration of low-frequency rTMS and fMRI to look at changes in network dynamics following inhibition of the left pcPM. Taken together, these methods allow us to begin to develop an understanding of the causal structure of motor networks during unimanual actions.

\section{METHODS}

\section{Participants}

Twenty-three neurologically healthy individuals (mean age $=29$ years, range $=19-48$ years; 10 men, all righthanded) were recruited from the University of California, Berkeley, community. The participants were screened for neuromuscular conditions that might preclude facile hand movements.

The institutional review board at the University of California, Berkeley, approved all protocols used in this study.

\section{Experimental Tasks}

Responses were made on five-key piano-style keyboards, with one keyboard positioned comfortably under each hand. For the imaging experiment, stimuli were displayed on a front-projecting display system. For the single-pulse TMS experiment, stimuli were presented on a computer monitor approximately 36 inches in front of the participant. E-Prime software (Psychology Software Tools, Sharpsburg, PA) was used to control the stimulus displays and collect the behavioral responses.

The experimental tasks were similar to that employed in a previous study (Verstynen et al., 2005, Experiment 2). Each trial began with a short cuing phase in which verbal instructions indicated the required hand and movement type for the forthcoming trial. At the top of the display, the target hand was cued by the word "Left" or "Right" and the movement type by the word "Simple" or "Complex." For the single-pulse TMS experiment, only left-hand movements were cued (see next section). Two rows of five horizontal lines were present at the center of the screen, and participants were instructed that these mapped to the five fingers of the specified hand. For simple movements, asterisks appeared over the corresponding finger positions of the upper row (thumb excluded) of the selected hand. In this condition, the participant was to simultaneously press and release the four finger keys in a repetitive fashion as fast as possible. For complex trials, the numbers 1 to 8 appeared over the lines, four on the upper row and four on the lower row. These indicated an eightelement sequence of responses. Two different sequences were used (1-3-2-4-2-3-4-1 or 3-2-4-1-4-1-3-2), each requiring two keypresses with each of the four fingers. As with the simple condition, the thumb was excluded and participants were instructed to cyclically produce the sequence as quickly as possible. The condition for a particular trial was selected at random.

Single-pulse TMS Experiment ( $n=15,5$ Men, Ages 19-29)

Experimental blocks consisted of eight simple and eight complex movement trials, all performed with the left hand and randomized within a block. Two different protocols were used (Figure 1A). For Group $1(n=8)$, the participants performed four blocks of trials, resulting in 


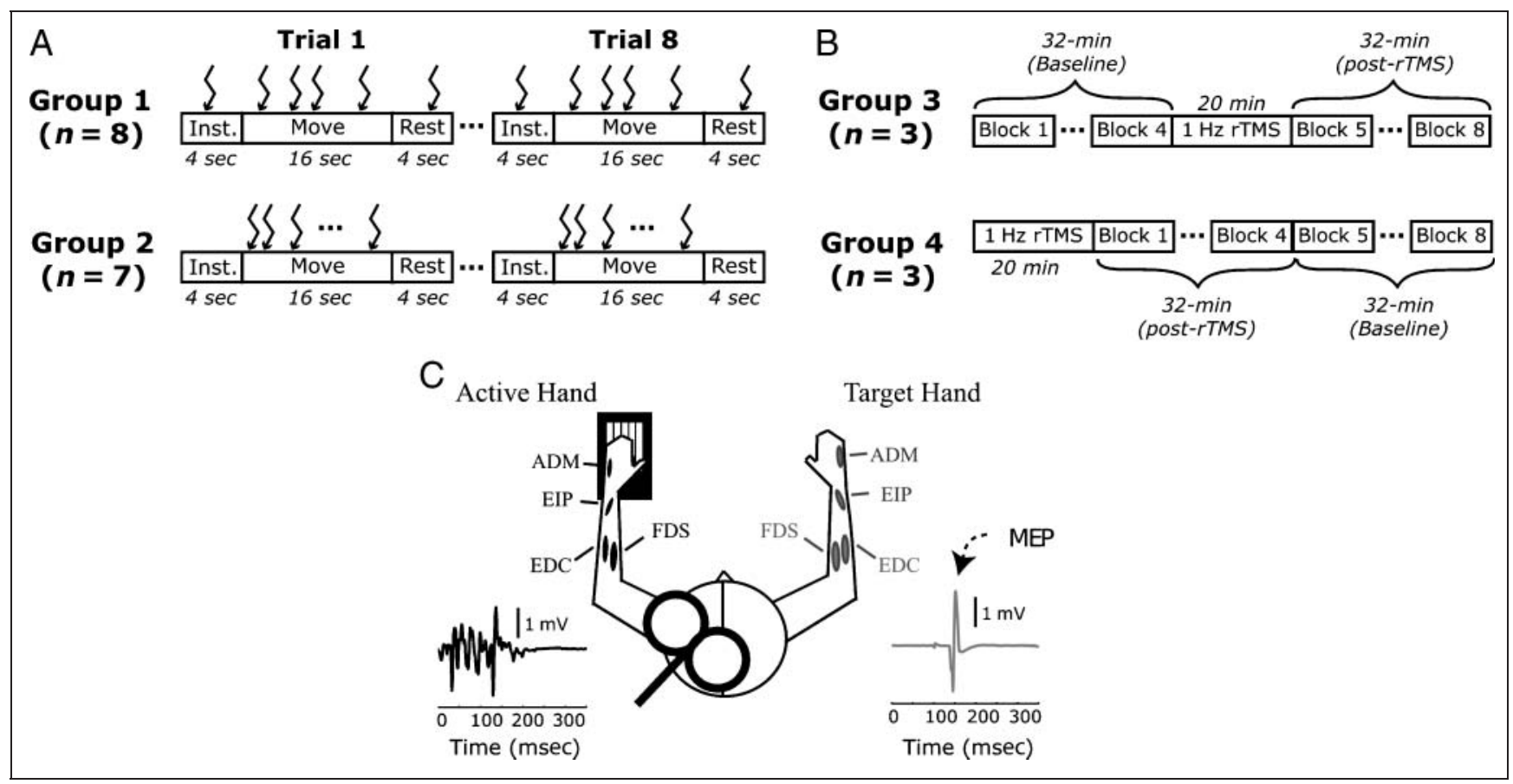

Figure 1. (A) Experimental design for the single-pulse TMS experiment. For Group 1, TMS pulses (squiggles) were applied during the instruction, movement, and rest phases of each trial. For Group 2, pulses were only applied during the movement phase. Each block consisted of eight trials ( 4 simple, 4 complex). (B) Experimental design for the rTMS/fMRI experiment. Stimulation order was counterbalanced across subjects. Group 3 completed the baseline phase before rTMS. Group 4 did all of the scanning blocks after rTMS, but the baseline phase was delayed until the rTMS effects had dissipated. (C) Experimental setup for the single-pulse TMS experiment. Responses were made with the left hand and TMS was targeted at left M1, producing MEPs in the stationary, right hand. Surface EMG was collected from three to four muscles in both hands.

32 simple and 32 complex movement trials. Six TMS pulses were applied during each trial: once during the instruction phase ( $4 \mathrm{sec}$ ), four times during the movement phase (16 sec), and once during the rest period ( $4 \mathrm{sec})$. Each pulse occurred at a random time within a 4 -sec window, with the constraint that there was at least $1 \mathrm{sec}$ between successive pulses. EMG data were stored for a 1-sec epoch beginning with the onset of each TMS pulse.

Group 2 participants $(n=7)$ completed 3 blocks of trials (24 simple, 24 complex). TMS pulses were applied only during the 16-sec movement phase and EMG was recorded continuously. The pulses were administered every 2-3 sec with the constraint that there was at least $1 \mathrm{sec}$ between successive pulses. We were thus able to increase the temporal sampling rate of TMS probes during the sequence of movements using this protocol.

\section{fMRI/rTMS Experiment ( $n=6,5$ Men, Ages 21-48)}

While in a supine position within the magnet, the response boards were placed on the participant's upper thighs. The scanning session consisted of two phases, a baseline phase and a post-rTMS phase. Within each phase, the participant completed four blocks of 32 trials each (2 trial types, Simple/Complex $\times 2$ Hands $\times 8$ Repetitions). Each block lasted approximately $8 \mathrm{~min}$ and there was a 1-min break between blocks. Trials within each block consisted of a 4 -sec instruction period, a 4-sec execution period, and an intertrial interval that ranged from 12 to $18 \mathrm{sec}$, with the exact value selected from an exponential distribution.

Two test orders were used, one in which the baseline phase preceded the post-rTMS phase and a second in which the baseline phase followed the post-rTMS phase. Three participants first completed the baseline phase (Group 3 in Figure 1B). They were then removed from the scanner and moved to an adjacent room for the application of 20 min of rTMS (see below). Immediately after rTMS, the participant was repositioned in the scanner and completed the four post-rTMS blocks. The other participants completed the baseline blocks after receiving rTMS (Group 4 in Figure 1B). These participants were initially positioned in the scanner in order to perform a set of diagnostic scans. They were then removed from the scanner and given rTMS in an adjacent room. Following this, they were repositioned in the scanner and completed all eight experimental blocks in succession. The first four blocks constituted the post-rTMS blocks and the last four blocks constituted the baseline block.

We recognize that obtaining baseline data after rTMS for Group 4 may be a concern. However, it was important to control for order effects in the fMRI data, given that the task-related BOLD signal can become smaller over time (Landau, Schumacher, Garavan, Druzgal, \& D'Esposito, 2004). A pilot study indicated that the effect of $20 \mathrm{~min}$ of rTMS on the BOLD response was absent after $30 \mathrm{~min}$. Given the 8 min duration of each block, plus the interblock break, 
the four baseline blocks for Group 4 were initiated approximately $36 \mathrm{~min}$ after rTMS.

\section{TMS Procedures}

TMS was performed with an air-cooled figure-of-eight coil (90 mm wing diameter) connected to a Magstim standard rapid stimulator (Magstim Co., Ltd., UK). Participants were fitted with a bouffant cap, secured to the scalp using surgical tape.

\section{Single-pulse TMS Study}

The TMS coil was placed over the left hemisphere, the hemisphere ipsilateral to the moving hand (and thus, contralateral to the stationary, right hand). The coil position was situated approximately $5-6 \mathrm{~cm}$ lateral to the vertex, $135^{\circ}$ to the mid-sagittal line so that the handle of the coil was angled in the posterior/ventral direction (Figure 1C). The coil location was adjusted to find the optimal scalp location for eliciting visible movement of the abductor digiti minimi (ADM) muscle of the right hand. Once identified, this location was marked on the cap.

To maximize sensitivity to detect changes in the MEPs, the stimulation intensity was determined by establishing a stimulus-response curve for each individual (Devanne, Lavoie, \& Capaday, 1997). Four TMS pulses (fixed 5-sec interstimulus interval) were delivered at a set of intensity levels. The first level was set at 35\% of the maximum stimulator output and increased in steps of 5\% until the amplitude of the MEPs recorded from the ADM muscle reached an asymptotic value. Once the maximum resting MEP amplitude was identified, stimulus intensity was decreased by steps of 5\% maximum stimulator output and four pulses were delivered. This procedure continued until the average MEP amplitude over the four pulses was half the maximum value. This stimulation level was used in the experiment. Note that at this value, MEPs were evident not only in the ADM but also in other muscles (see below).

\section{Repetitive Stimulation}

A frameless stereotaxic localization system (Brainsight; Rogue-Research, Montreal, Canada) was used to identify the pcPM target location for r'TMS. The targeted pcPM area in the left hemisphere was estimated using functional localizers obtained in a prior fMRI session. Of the six participants in the rTMS/fMRI study, two had been tested in a previous study using a similar paradigm (Verstynen et al., 2005). The other four participants underwent an initial scanning session 1 to 3 weeks before testing. During this session, they completed 4 blocks of trials (32 trials for each of the four conditions). Ipsilateral pcPM was identified as those voxels which were (a) active during ipsilateral hand movements ( $t>3.5)$, (b) modulated by task complexity, and (c) shifted in an anterior-lateral direction from the peak of activation in precentral voxels during contralateral hand movements. Functional images highlighting the selected regions of interest (ROIs) were loaded into the stereotaxic system and overlaid on a 3-D reconstruction of the participant's brain. Once identified, the scalp location of this region relative to the vertex was marked on the cap.

We also performed single-pulse TMS during the prescanning localization session to identify the left hemisphere motor threshold. The motor threshold was defined as the stimulation intensity required to elicit MEPs on 5 out of 10 consecutive pulses in the resting contralateral first dorsal interosseous. Threshold measurements were limited to the right hand, with the coil positioned over the left hemisphere. The coil was oriented $135^{\circ}$ to the mid-sagittal plane, an angle that provides optimal stimulation of neurons lying parallel along the medial bank of the precentral gyrus (Nagarajan, Durand, \& Hsuing-Hsu, 1997). A minimum amplitude of $0.5 \mathrm{mV}$ peak-to-peak on the unrectified EMG signal was used as the criterion for scoring the presence of an MEP.

During the rTMS/fMRI session, the participant was refitted with the cap in the control room. The location of the vertex was identified relative to fiducial markers. The optimal stimulation site for left pcPM was then identified relative to the vertex. There were 1200 TMS pulses delivered at a rate of $1 \mathrm{~Hz}(20 \mathrm{~min}$ ) over the target location. For this stimulation, the coil was oriented $45 \%$ to the midsagittal plane. The intensity was set at $115 \%$ of the motor threshold.

\section{Electromyography and MEP Analysis}

Electromyographic (EMG) activity was recorded by bipolar surface electrodes connected to an eight-channel Bagnoli amplifier (Delsys, Boston, MA), digitized at a rate of $2 \mathrm{kHz}$ (National Instruments, Austin, TX). The recordings were made from a set of targeted muscles from the moving (left) and stationary (right) hands (Figure 1C).

For the Group 1 participants, EMG was recorded in four pairs of homologous muscles ( 8 muscles total): an intrinsic hand muscle (abductor digiti minimi, ADM), two extrinsic extensor muscles (extensor indicis proprius, EIP; extensor digitorum communis, EDC), and an extrinsic flexor muscle (flexor digitorum superficialis, FDS). Each recording was time-locked to the TMS pulse and lasted for 1 sec. We did not obtain EMG data prior to the TMS pulses for this group because the original software used for data collection did not allow for pre-TMS triggers in the EMG recording process.

For the Group 2 participants, EMG was recorded continuously and two input channels were used to log the timing of the TMS pulses. Although this precluded recording EMG in the left and right FDS, it allowed us to obtain background EMG data in the targeted muscles. Background EMG was calculated taking the root-mean-squared (RMS) signal recorded for $20 \mathrm{msec}$ prior to the TMS pulse. The background EMG was monitored visually throughout the experiment. If the amplitude of the background signal 
exceeded $10 \%$ of the maximum voluntary contraction for more than $2 \mathrm{sec}$ in any single trial, the data for the entire block were discarded and that block was repeated. This only occurred for one participant (2 blocks).

MEP amplitude served as the primary dependent variable for the TMS studies. These were calculated as the maximum peak-to-peak difference amplitude in the unrectified EMG signal occurring within a window of 1550 msec after the TMS pulse. Given the relatively small number of factors being compared, paired-sample $t$ tests were employed to evaluate changes in MEPs and EMG spectral density between the simple and complex movement conditions. In order to determine the relationship between EMG activity in the moving left hand and MEPs evoked in the stationary right hand, we used a correlation analysis. We first calculated the RMS EMG activity from the sensors over each muscle in the moving hand. The RMS EMG activity, spanning a window from $400 \mathrm{msec}$ before to 400 msec after each TMS pulse was identified and binned into 40 segments (20 msec each). Correlations were then calculated between the EMG activity in each bin and the MEP values using Spearman's rank-order correlation test. The consistency of the set of correlations at each bin was estimated across subjects using a onesample $t$ test.

\section{fMRI Procedures and Analysis}

Scanning was performed with a Varian 4-T Unity INOVA system. A total of 1944 functional volumes were acquired across eight scans using an echo-planar imaging (EPI) pulse sequence sensitive to BOLD changes (EPI parameters: 18 slices interleaved, TR $=2000 \mathrm{msec}$, TE $=28 \mathrm{msec}$, matrix size $=64 \times 64$, thickness $=3 \mathrm{~mm}$, gap $=0.5 \mathrm{~mm}$, yielding isotropic voxels of $3.5 \mathrm{~mm}$ ). The field-of-view $(22.4 \times 22.4 \times 6.3 \mathrm{~cm})$ for these images encompassed all cortical regions above the Sylvian fissure. The onset of each functional scan was synchronized to the onset of each task-relevant event, including the instruction and imperative stimuli, as well as the onset of the rest periods. In addition to the functional scans, a high-resolution T1weighted anatomical image was acquired using a FLASH pulse sequence $(91$ slices, matrix size $=91 \times 109$, thickness $=2 \mathrm{~mm}$ ).

All images were reconstructed from $k$-space using local software. Functional data were analyzed using SPM2 (Wellcome Institute of Cognitive Neurology, London, UK). The EPI images for each participant were corrected for differences in the slice acquisition time, and realigned to the first image in the series. All images were then smoothed using a 4-mm isotropic Gaussian kernel. Given that we had insufficient signal-to-noise from the functional volumes to use automated coregistration routine, all functional images were reoriented manually to match the coordinate frame of the anatomical T1 image. Images were simply coregistered but not resliced and all analyses were performed in EPI space.
Estimation of task-related changes was performed using the general linear model (GLM; see Kiebel \& Holmes, 2004). Separate parameter estimates were determined for each of the four movement conditions (simple or complex movements produced with left or right hands). Estimated head-movement parameters, obtained from the realignment process, and block effects were also included as covariates in the estimation of the GLM. The wholebrain statistical maps in this space were used to functionally identify M1 in each subject using the Marsbar toolbox in SPM2 and custom written software. For graphical purposes, the statistical maps were resliced and coregistered to an individual's T1 anatomical images, although all analyses were performed in the EPI coordinate frames.

Two diamond-shaped ROIs (10.5 mm diameter; 7 total voxels) were identified in each hemisphere (4 ROIs total), one in M1 and the other in pcPM. The location of the M1 ROI was determined by identifying the voxel on the posterior bank of the precentral gyrus, including the central sulcus, which showed the highest level of activity during contralateral hand movements. The pcPM ROI was defined relative to this point, with the initial location set $2 \mathrm{~cm}$ away in the ventral-lateral direction along the precentral gyrus (Hanakawa et al., 2005; Verstynen et al., 2005). The final position of this ROI was manually adjusted on an individual basis to accommodate for the descending angle of the participant's precentral gyrus. The primary fMRI analyses were restricted to voxels in these ROIs.

Single-trial evoked responses were estimated by averaging the time series across all voxels within an ROI and examining changes in the BOLD signal for the first $24 \mathrm{sec}$ after trial onset. The evoked responses for each trial were interpolated to estimate a sampling rate of $1 \mathrm{~Hz}$ and smoothed using a moving-average box-car filter ( 4 sec width). These responses were normalized to the mean voxel value for each block. Mean task-related responses were estimated by averaging the evoked responses across all trials. For the group analysis, the peak average evoked response between 7 and $14 \mathrm{sec}$ after trial onset was used as an estimate of response magnitude for each of the four movement conditions.

Connectivity analysis between ROIs was performed using simple correlation methods. On a trial-by-trial basis, the peak evoked response from each M1 ROI was correlated using a nonparametric method (Spearman's rank-order correlation coefficient). This method provides an estimate of functional connectivity between the two motor cortices. Single-subject confidence intervals and statistical significance were determined using sampling-with-replacement permutation tests, that is, by bootstrapping (see Results; Henderson, 2005). For each hand, movement type, and stimulation condition (i.e., post-rTMS or baseline), we permuted the single-trial BOLD values from left and right M1. This generated a simulated "chance" distribution that was used to compare the true observed correlation value and estimate statistical significance on a per subject basis. 


\section{RESULTS}

\section{Unimanual Movements and Ipsilateral M1 Excitability}

We used single-pulse TMS to directly examine changes in ipsilateral cortical excitability during simple and complex movements. TMS was applied over the hand region of M1 in the left hemisphere (Figure 1C) while participants produced movements with the ipsilateral left hand.

The TMS pulses did not disrupt performance of simple and complex movements. Participants had little difficulty producing the synchronized movements in the simple condition and, overall, less than 5\% of the responses in the sequence condition were performed out of order. Movement rates for the simple and sequence conditions were statistically equivalent $[t(14)=0.94, p>.05]$. On average, participants produced 60 repetitions of the four-finger tapping movement during the simple task (mean interkeypress interval $=278 \mathrm{msec}, S E= \pm 17 \mathrm{msec}$ ) and completed 10 cycles of the sequence during the sequence task (mean $=265 \mathrm{msec}, S E= \pm 16 \mathrm{msec}$ ).

Overall MEP amplitudes in the stationary right hand were larger when the participants produced complex movements with the left hand compared to simple movements with the left hand (Figure 2). This effect was only significant during the movement phase [movement: $t(15)=3.59, p<$ .01 ; instruction: $t(7)=1.46, p>.05$; rest: $t(7)=1.82, p>$ .05]. ${ }^{1}$ The MEP amplitude increased by a mean of $39 \%$ during complex movements over that observed during simple actions. This effect was largest in the intrinsic hand muscle (ADM), although similar effects were observed in the more proximal, extrinsic hand muscles (EIP, EDC, and FDS). Background EMG levels were recorded in a subset of the participants (Group 2) and did not show a consistent change between the complex and simple conditions (all $p s>.05$ ).

Group 2 also received more TMS pulses during the movement period than Group 1, allowing for enough power

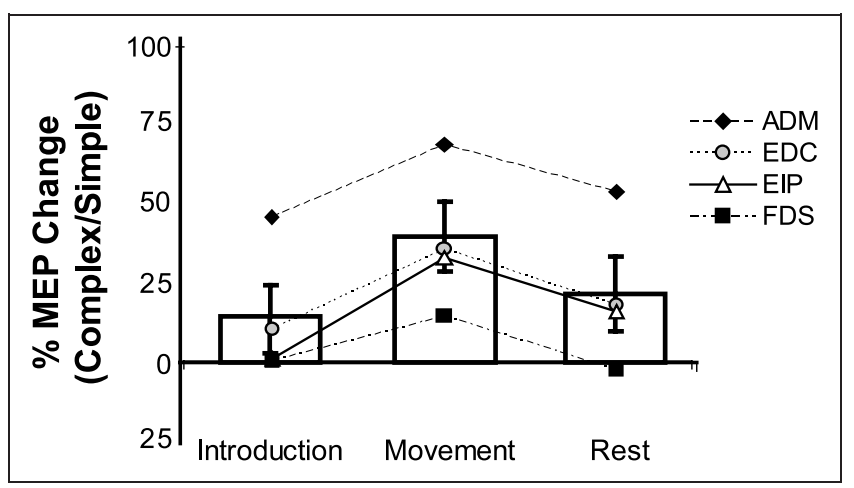

Figure 2. Percent change in right-hand MEPs during complex left-hand movements compared to simple left movements. The white bars show the mean changes across all muscles (error bars represent standard error across participants). The four lines indicate the change value for each of the four muscles. to ask how the excitability changes in ipsilateral, left M1 were influenced by the state of the moving hand. To quantify this relationship, we analyzed the EMG data over an epoch spanning 400 msec on either side of the TMS pulse, tabulating the rectified EMG level in 20-msec bins. On each trial, the amplitude of this binned EMG signal for a given left-hand muscle was compared with the TMS-elicited MEP amplitude recorded in the homologous right-hand muscle for that trial. Correlating these measures across trials provided an estimate of the temporal relationship between muscle activity and ipsilateral M1 excitability (Figure 2A-C). For the most distal hand muscle, ADM, the correlation was largest during the complex movements prior to the onset of the TMS pulse. In contrast, the extensor complex (EDC) showed the opposite pattern: Here, the correlation was greater during simple movements compared to complex movements. ${ }^{2}$ Moreover, the temporal extent of the correlation for EDC was shorter than that observed in the ADM, beginning about $60 \mathrm{msec}$ before TMS onset. EIP did not show a significant positive correlation between the state of the moving hand and MEP amplitudes during either simple or complex actions.

The inconsistent pattern of correlations may be related to the relative engagement of the muscles during the simple and complex movements. To evaluate the activity of each muscle during the simple and complex movements, we calculated the power spectrum of EMG activity in the moving hand across the entire 16-sec movement period. The power density function then was averaged across frequencies in the range of 1 to $29 \mathrm{~Hz}$, a range that spans both the movement frequency and motor unit firing frequencies (Beck et al., 2007). As can be seen in Figure 3D, the muscles were not equally activated across the two movement conditions. The ADM was active more during complex than during simple movements $[t(6)=2.41$, $p<.05]$. In contrast, the effect was reversed for the EDC: This muscle was more active during simple movements $[t(6)=3.35, p<.01]$. Activity in the EIP was weak for both conditions and not significantly modified by movement type $[t(6)<1]$. When considered along with the correlation results, it appears that the modulation of the MEPs is greatest in muscles that are most active for a particular task.

\section{Hemodynamic Responses in Precentral Motor Areas}

To visualize the cortical dynamics present during these unimanual movements, we used fMRI to look at the hemodynamic changes in cortical motor areas during both left- and right-hand actions. Our initial analysis was restricted to baseline blocks, scanning runs in which the hemodynamic response was not influenced by TMS. For half the participants, these blocks were performed prior to rTMS. For the other half, these blocks were performed at least 35 min after rTMS, at a time when the effects of rTMS had dissipated. 

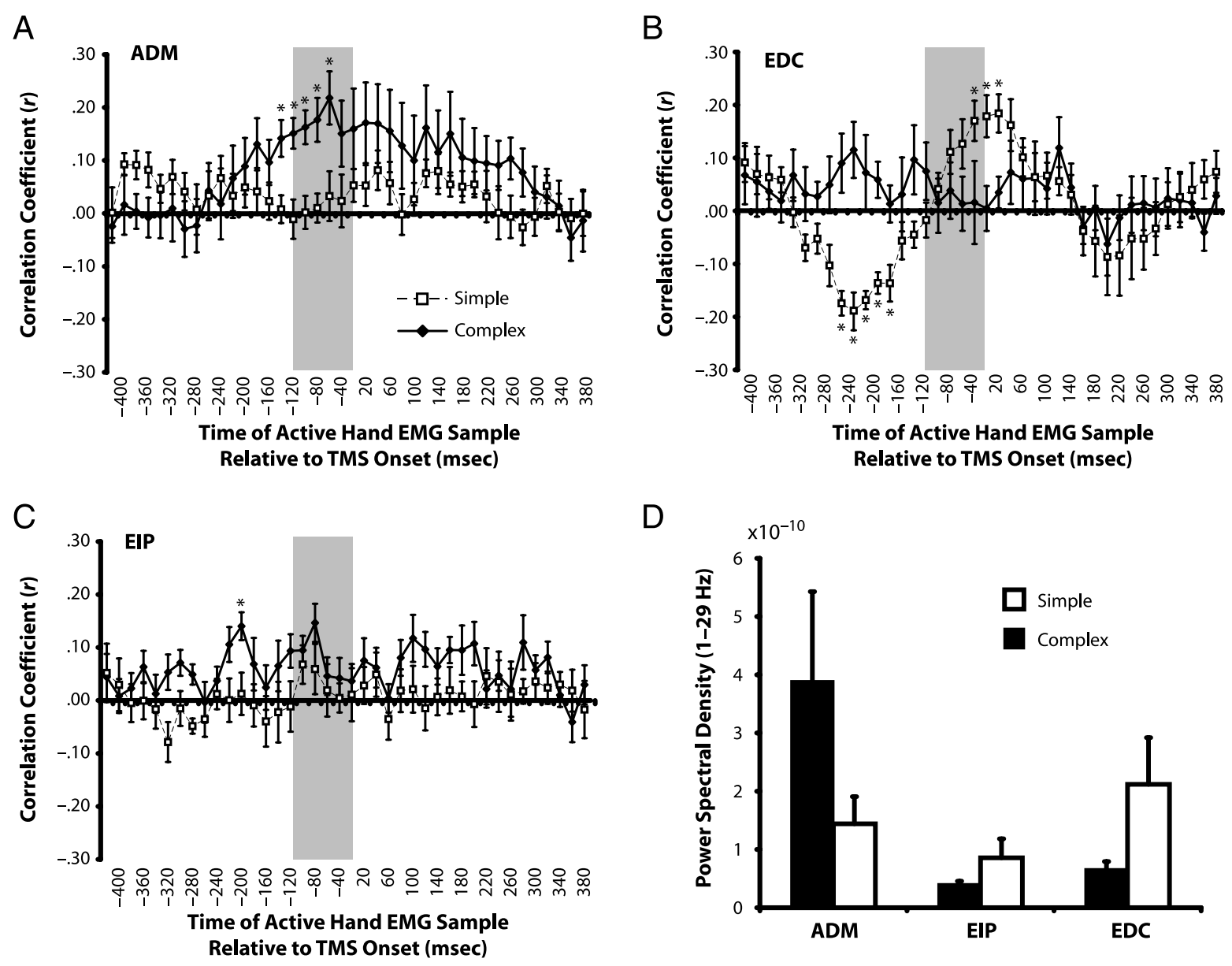

Figure 3. (A-C) Cross-correlations between EMG state in the moving left-hand and the size of the MEP in the homologous right-hand muscle, plotted as a function of the time of the TMS pulse. Filled symbols represent values for complex trials and open symbols represent simple trials. Error bars represent standard error. The gray rectangles highlight a window $100 \mathrm{msec}$ before the TMS pulse. Asterisks indicate significant correlations across subjects $(p<.05)$. (D) Mean power spectral density of the EMG from muscles of the moving left hand during simple and complex movements.

We first focused on the contrast of complex movements versus rest, analyzing the data separately for the left- and right-hand conditions. The center of activation in the contralateral hemisphere was squarely in the central sulcus and corresponds well with Brodmann's area 4. We also observed activation in caudal regions of the ipsilateral precentral gyrus. Replicating previous findings (Hanakawa et al., 2005; Verstynen et al., 2005; Cramer et al., 1999), the center of this activation was shifted $2 \mathrm{~cm}$ in an anterior-lateralventral direction from that observed during contralateral hand movements. Figure 4A shows a representative statistical map for one participant when performing the complex movements with either the left or right hands. The premotor region that was active during ipsilateral hand movements (blue arrow for left-hand movement) falls on the medial bank of the precentral gyrus, corresponding to the posterior aspect of Brodmann's area 6.

To look at task-related responses in these two areas, we adopted an ROI approach. Figure 4B shows the location of the M1 and pcPM ROIs and the trial-averaged evoked responses for the same participant. The response profiles were markedly different for the two ROIs (Figure 4C). A main effect for hand was observed in left M1 [hand: $F(1$, $7)=278.74, p<.001$ ] and right $\mathrm{M} 1$ [hand: $F(1,7)=$ $19.81, p=.003$ ], reflecting the fact that the response in these regions was much greater during movements of the contralateral hand. Neither M1 region showed a significant main effect of movement complexity or a significant Hand $\times$ Task interaction (all $F_{\mathrm{S}}<1$ ).

In contrast, the left pcPM region was strongly activated during movements with either the right or left hands. Although this response was stronger for contralateral hand movements [hand: $F(1,7)=8.29, p=.024$ ], the effect was modulated by movement complexity [task: $F(1,7)=$ 5.92, $p=.045]$ for both hands (Hand $\times$ Task: $F<1$ ). The ipsilateral response was weaker in right pcPM and was not modulated by movement complexity [task: $F(1,7)=$ $1.15, p=.32]$. This region did show an effect of hand $[F(1,7)=8.37, p=.023]$, similar to what was observed in the adjacent right M1 region. The Hand $\times$ Task interaction was also not significant for right pcPM (Hand $x$ Task: $F<1$ ). The asymmetrical pattern observed here, with ipsilateral activation more pronounced during left-hand movements (and thus, in the left hemisphere), is consistent 
with previous reports (Verstynen et al., 2005; Kobayashi et al., 2003; Kawashima et al., 1997; Kim et al., 1993).

Although we did not observe a reliable increase in the M1 BOLD response during ipsilateral movements, an evoked response could be observed in some trials (Figure 5A). The magnitude of this ipsilateral activation appeared to be correlated with the degree of activation in M1 of the other hemisphere, the one contralateral to the moving hand. This observation suggested a degree of mirror activity in the M1 regions of the two hemispheres. To evaluate this hypothesis, we performed a trial-by-trial correlation of the evoked responses in the M1 ROIs. An example from one participant is shown in Figure $5 \mathrm{~B}$ and the correlation values for each participant in the four movement conditions are shown in Figure 5C. During right-hand movements, these correlations were significantly greater than zero [simple: $t(7)=3.40, p<.01$; complex: $t(7)=5.12, p<.005]$.
The correlations were weaker during left-hand movements, with the effect only being reliable for the complex condition $[t(7)=2.65, p<.025$; simple: $t(7)=1.84, p>.05]$. Thus, the results of a more sensitive analysis of the hemodynamic response in M1 are consistent with the mirroring of M1 activity observed in the single-pulse TMS experiment. Of particular interest is the observation that the degree of correlation between M1 responses changes in the same way as the activation observed in the left pcPM ROI. This suggests that the left pcPM may be playing some role in the modulation of M1 mirroring.

\section{Network Changes following Functional Deactivation of the Left Precentral Premotor Area}

To assess the relationship between pcPM and coupling of the two primary motor cortices, we used 1-Hz rTMS to

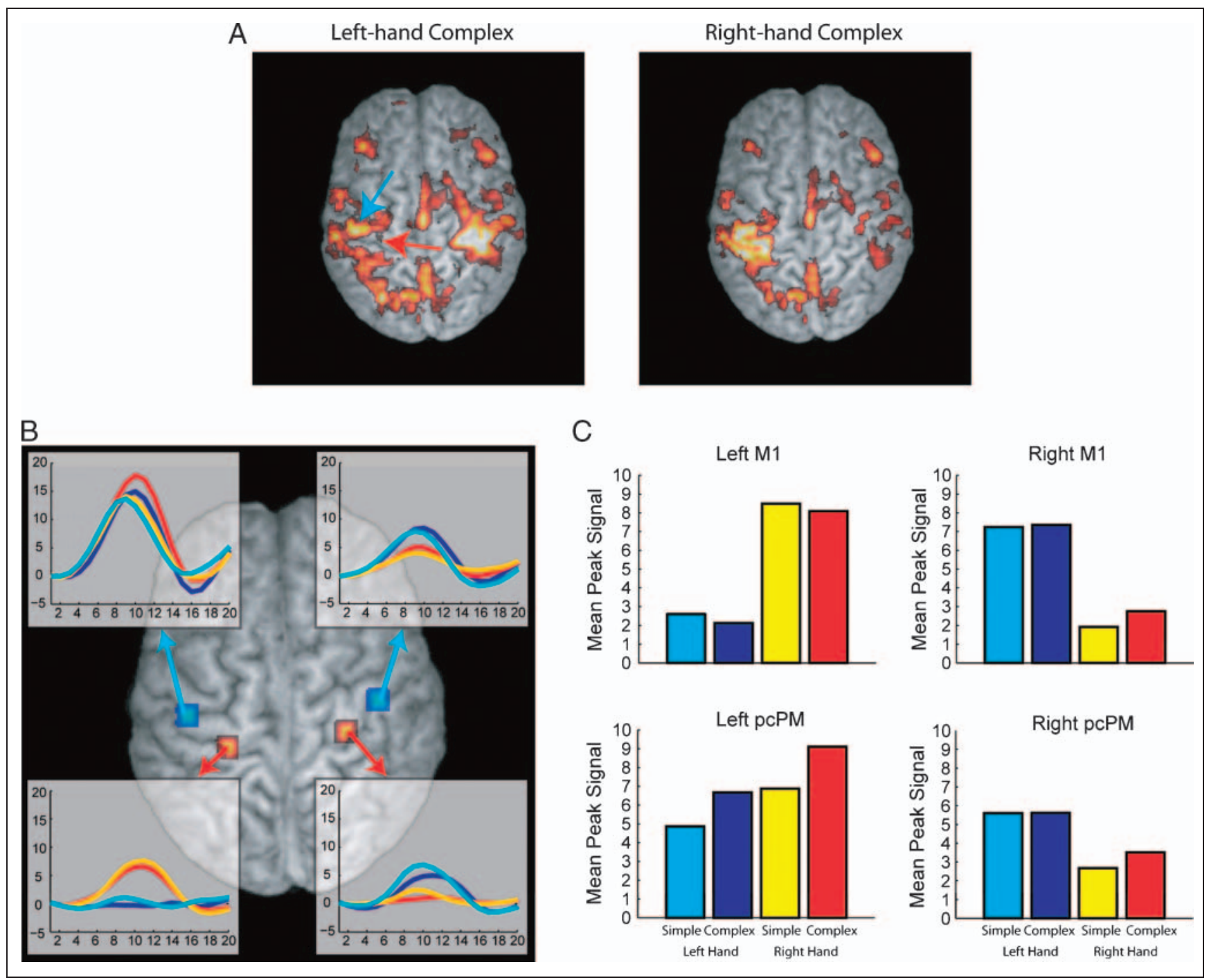

Figure 4. (A) Statistical map of active voxels for the complex movement conditions for a single subject (move-rest, $t>3.5$ ). Blue arrow indicates cluster of ipsilateral activity in the precentral gyrus of the left hemisphere. Red arrow shows peak of activity during contralateral (right) hand actions. (B) Trial-average BOLD response in pcPM (blue) and M1 (red) ROIs for the subject shown in A. (C) Group-average responses for each ROI. Color scheme for B and C is: left simple (light blue), left complex (dark blue), right simple (yellow), and right complex (red). 


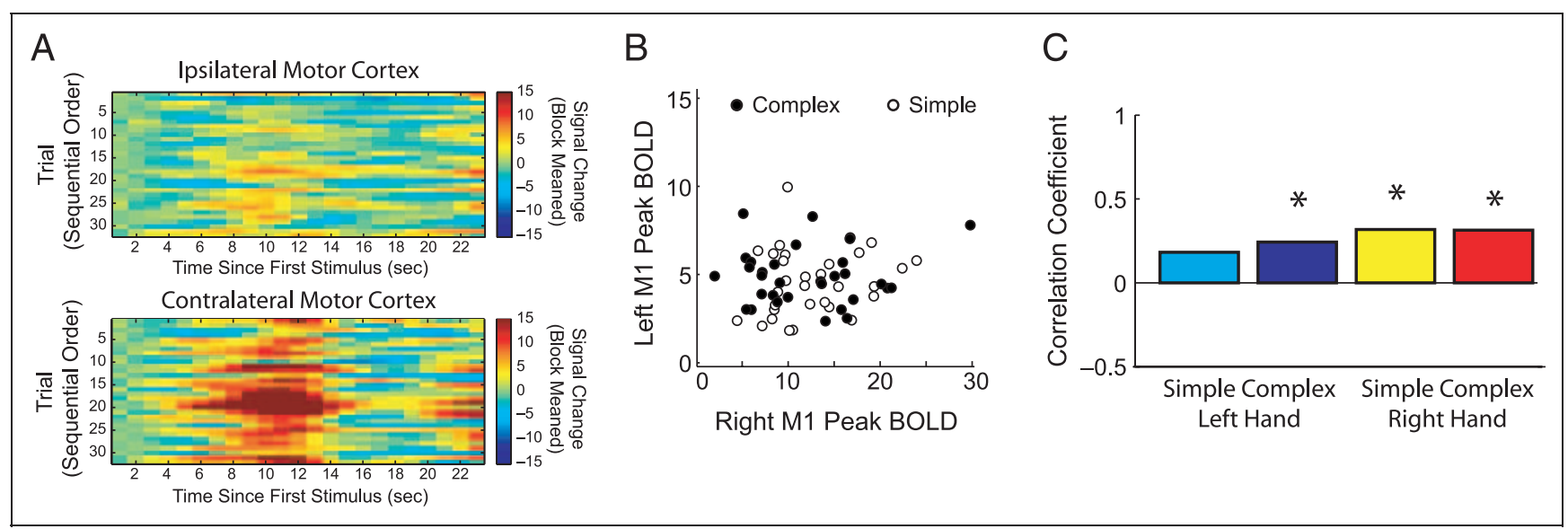

Figure 5. (A) Heat maps of single-trial responses in the left and right M1 ROI for a typical subject. (B) Correlation of peak evoked responses across trials for the subject shown in A. (C) Group-averaged correlation values across all four movement conditions. Asterisks indicate $p<.05$. Same color scheme as Figure 4C.

transiently perturb activity in left pcPM. This rTMS protocol has been shown to reduce neural activity in the underlying cortical region, presumably by reducing the metabolic resources available to stimulated cells and disrupting local synchrony (Pasley, Allen, \& Freeman, 2009; Allen, Pasley, Duong, \& Freeman, 2007). The direct effects of $1 \mathrm{~Hz}$ rTMS have been estimated to affect a $1-2 \mathrm{~cm}^{2}$ area of cortex (Esselle \& Stuchly, 1992).

For each participant, fMRI data were obtained during a baseline phase and post-rTMS phase. The order was counterbalanced across participants. For participants tested in Group 4, a minimum interval of 36 min separated the end of the rTMS and the start of baseline measurements. In pilot studies (not shown), we found that $20 \mathrm{~min}$ of $1 \mathrm{~Hz}$ rTMS led to a reduction of the BOLD response in the targeted voxels for no longer than 30 min after stimulation.

Figure 6A shows the peak evoked response in the four ROIs following left pcPM rTMS, relative to baseline. Consistent with the presumed effect of rTMS on cortical excitability, a consistent decrease in the BOLD response was observed in the left pcPM ROI. The effect was most pronounced during contralateral, right-hand movements [simple: $t(5)=5.38, p<.005$; complex: $t(5)=2.96, p<.025$ ], but it was also significant during ipsilateral, left-hand movements [simple: $t(5)=2.57, p<.025$; complex $t(5)=2.09$, $p<.05]$.

Activation in the adjacent M1 region was also attenuated. This effect was only reliable during complex righthand movements $[t(5)=3.45, p<.01]$, although a similar
Figure 6. Mean percent change in peak amplitude of task-related BOLD, following rTMS over left pcPM for each ROI. Asterisks indicate $p<.05$.

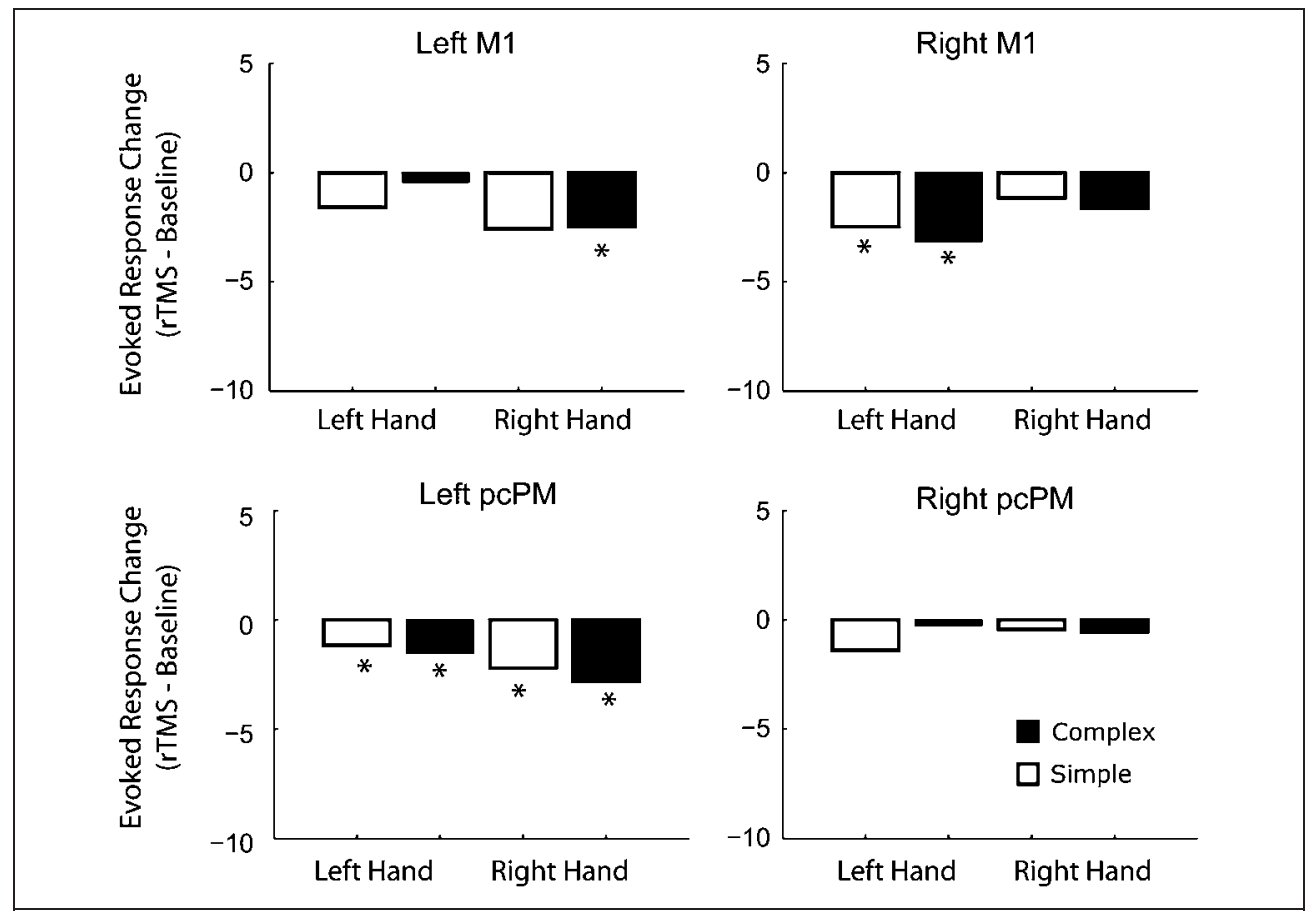




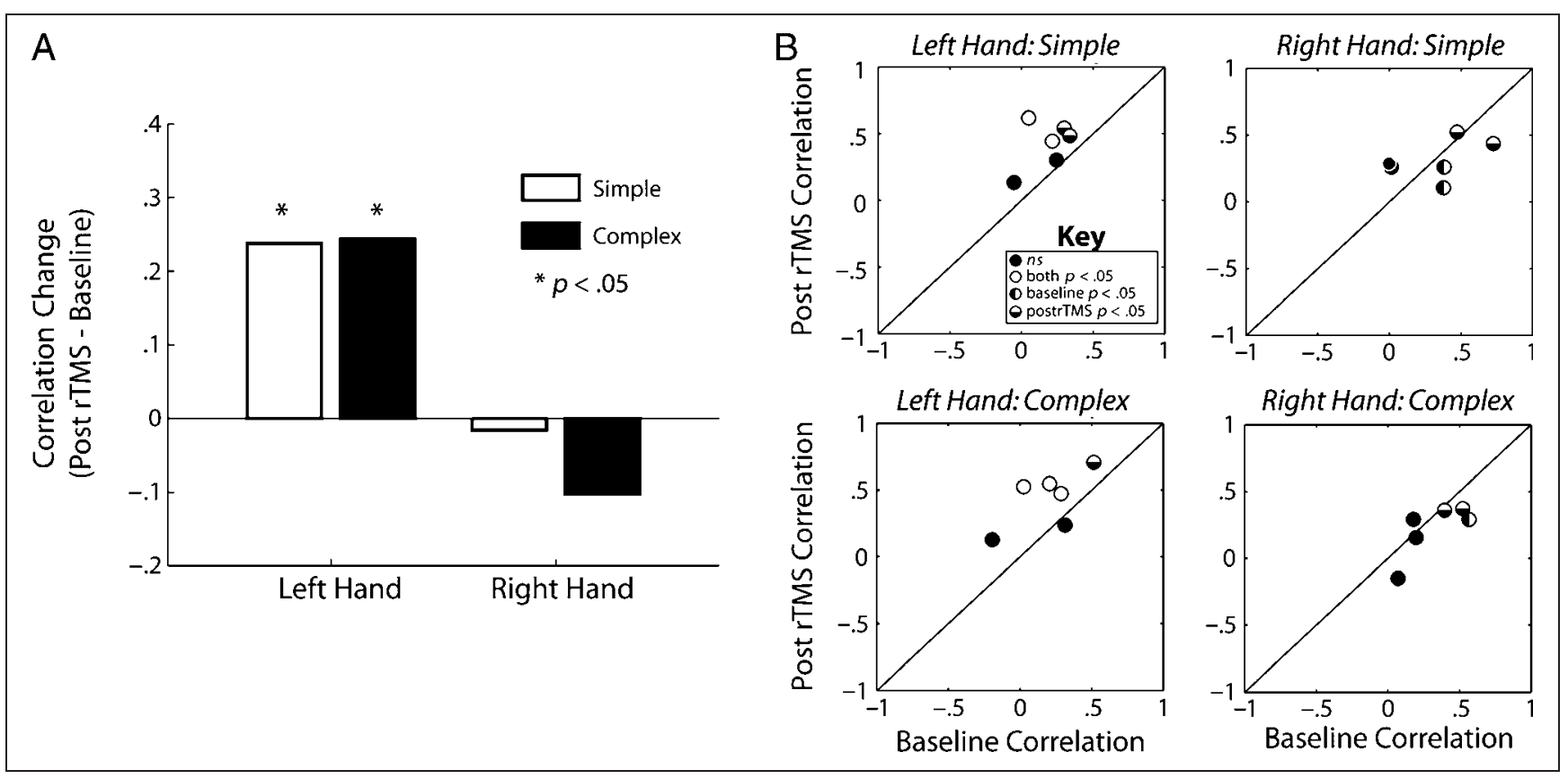

Figure 7. (A) Mean changes in left and right M1 activity correlations following rTMS. Asterisks indicate significant correlations $(p<.05,1$-sample $t$ tests). (B) Individual subject comparison of results in A for each movement condition. Data points above the identity line indicate increased correlations after rTMS and data points below the line indicate decreased correlations.

trend was observed in the simple movement condition $[t(5)=1.95, p<.10]$. The BOLD response in left M1 during ipsilateral movements was not affected by rTMS of left pcPM [simple: $t(5) 1.24, p>$.10.; complex: $t(5)=$ $0.87, p>.10]$. Thus, although rTMS led to a consistent reduction in activation of the targeted premotor region, the effect in adjacent, left M1 was limited to conditions in which movements were produced with the contralateral hand. It should be noted that the BOLD response in M1 was weak during ipsilateral movements in the baseline condition (see Figure 4C); thus, there is little signal to be attenuated.

The activation pattern in right M1 was also influenced by rTMS of left pcPM. Similar to what was observed in left M1, the BOLD response in right M1 was reduced during contralateral, left-hand movements. This effect was similar for both movement conditions [simple: $t(5)=3.18, p<$ .025 ; complex: $t(5)=2.75, p<.025]$. Activation during ipsilateral, right-hand movements was not affected by rTMS of left pcPM [simple: $t(5)=1.30, p>.10$; complex: $t(5)=1.40, p>.10]$. However, we did not observe any changes in the BOLD response of right pcPM following rTMS of the homologous region in the left hemisphere (all $p s>.05$ ).

In addition to the changes in the mean hemodynamic response within each M1, we also examined how rTMS influenced the correlation of the activation patterns in the two primary motor areas. To this end, we performed trial-by-trial correlations between left and right M1 activity during the post-rTMS blocks. As reported above, these values were positive during the baseline blocks. Following rTMS, we observed a change in these correlations, but only during left-hand movements (Figure 7A). For both simple and complex movements, the correlations were higher in the post-rTMS blocks compared to the baseline blocks [simple: $t(5)=3.34, p<.02$; complex: $t(5)=3.05$, $p<.03]$, that is, the positive correlation between the responses in left and right motor cortex became greater during left-hand movements following rTMS of left pcPM. In contrast, during right-hand movements, the degree of coupling between the two M1 regions was unaffected by rTMS [simple: $t(5)<1$; complex: $t(5)=-1.75, p>.05$ ]

To illustrate the saliency of these correlation effects on the single-subject level, we adopted a permutation test approach (Figure 7B). In all but two subjects, we observed a positive and significant correlation during left-hand actions following rTMS. In some cases, the M1 correlation did not reach statistical threshold until after rTMS was applied. More importantly, the cluster of correlation points is consistently above the identity line, illustrating that rTMS consistently increased the mirroring between the two M1s during left-hand actions. A less consistent pattern was observed during right-hand actions, with no consistent change following rTMS.

\section{DISCUSSION}

Activation of ipsilateral precentral motor areas has been observed consistently in functional imaging studies (Hanakawa et al., 2005; Kobayashi et al., 2003; Cramer et al., 1999; Singh et al., 1998; Kawashima et al., 1997; Kim et al., 1993; Shibasaki et al., 1993), an effect that is pronounced especially in the left hemisphere during the production of 
complex movements with the left hand (Verstynen et al., 2005). We tested the hypothesis that this ipsilateral activity reflects a mirroring of the motor commands being executed by the contralateral hemisphere. Through the converging and combined use of TMS and fMRI, we observed an increase in coupling of the two motor cortices during complex unimanual movements. In addition, our results suggest that activity in left premotor cortex during ipsilateral hand actions may be associated with a mechanism that prevents the expression of unwanted mirror movements in the contralateral right hand. These findings provide several novel insights into the dynamics of motor networks during unimanual movements.

The imaging data confirm previous observations (Hanakawa et al., 2005; Verstynen et al., 2005) that the locus of ipsilateral activity is centered in the premotor region of the precentral gyrus and not in primary motor cortex. Moreover, we replicated our previous observation that responses in the pcPM region of the left hemisphere vary with movement complexity, with the ipsilateral activation of this region greater for more complex movements. This variation is sufficiently large to be detected with traditional GLM analysis methods. A similar pattern was not evident in right pcPM. This hemispheric asymmetry has been reported in many studies (Kobayashi et al., 2003; Singh et al., 1998; Kawashima et al., 1997; Kim et al., 1993; Rao et al., 1993; Shibasaki et al., 1993) and is likely related to left hemisphere contributions to high-level motor planning and control (Johnson-Frey, Newman-Norlund, \& Grafton, 2005). Although the complex task used here, sequential finger movements, places an additional temporal structure on control systems, other results suggest that qualitatively similar asymmetries arise when complexity increases without this additional temporal structure (e.g., chord-like hand movements; Verstynen et al., 2005).

We did not detect a significant increase in the BOLD response in primary motor cortex during ipsilateral movements. Nonetheless, converging lines of evidence suggest that excitability in primary motor cortex was modulated during ipsilateral actions. First, the amplitude of TMSinduced MEPs in the right hand (assessed by left M1 stimulation) was modulated by the type of movement produced by the left hand: MEPs in the stationary hand were larger when the left hand produced complex actions. Second, the temporal dynamics of M1 excitability changed in a muscle-specific manner: MEPs elicited in right-hand muscles varied as a function of the specific type of movement being produced with the left hand, with the largest increases observed when the homologous muscle was active. Whereas the modulation of MEPs can be mediated at cortical and spinal levels (Duque, Lew, Mazzocchio, Olivier, \& Ivry, 2010), a more fine-grained analysis of the fMRI data indicated that activity in M1 was modulated during ipsilateral movements. Trial-by-trial fluctuations in the BOLD response of ipsilateral M1 were correlated with the amplitude of the BOLD response in the contralateral M1 (see also Biswal et al., 1995). Taken together, these results point to a weak, but consistent, coupling of activity between left and right M1 during unimanual actions.

The correlation between the two motor cortices increased during complex movements, similar to what is found in ipsilateral pcPM responses, suggesting that these two phenomena may be functionally linked. We directly tested this hypothesis by combining low-frequency rTMS with fMRI. Stimulation of the left pcPM produced a local decrease in the BOLD response from pcPM, as well as in adjacent left $\mathrm{M} 1$ and contralateral motor areas. This overall change in BOLD between regions confirms that both motor cortices are functionally connected to pcPM (Bestmann, Baudewig, Siebner, Rothwell, \& Frahm, 2003). Despite this overall decrease in BOLD signal, the correlations between left and right M1 activity during left-hand movements increased following rTMS of the left pcPM. This dissociation suggests that left pcPM may regulate the degree of coupling between the two motor cortices. During complex actions, an upstream motor area involved in motor planning may lead to bilateral activation of M1 (Stinear et al., 2001; Muellbacher et al., 2000; Stedman et al., 1998). The pcPM area may suppress the expression of unwanted mirror movements by directly inhibiting ipsilateral M1.

Further support for this inhibitory control hypothesis comes from several previous fMRI (e.g., Hayashi et al., 2008) and TMS (e.g., Duque et al., 2007) studies of the cortical dynamics during movement. This work has shown a suppression of activity/excitability in M1 during the preparation and execution of an ipsilateral movement. However, when the movements are complex, there is an increase in bilateral activation (e.g., Verstynen et al., 2005), and correspondingly, the suppression of M1 is attenuated (Hayashi et al., 2008), at least in the left hemisphere. Inhibitory mechanisms (Duque et al., 2010; Gilio, Rizzo, Siebner, \& Rothwell, 2003; Ferbert et al., 1992) may be required to ensure that bilateral representations of action plans do not result in involuntary actions of the nonmoving hand.

Such mechanisms may involve interhemispheric interactions. For example, TMS studies of transcallosal inhibition have shown that the left hemisphere exhibits a stronger inhibitory influence on right M1 compared to the inhibitory effects of the right hemisphere on left M1 (Baumer et al., 2007; Netz, 1999; Netz, Ziemann, \& Homberg, 1995). Our findings indicate that, along with these interhemispheric inhibitory control mechanisms, intrahemispheric inhibitory mechanisms may also be at play (see also Duque \& Ivry, 2009). When left hemisphere pcPM was disrupted by rTMS, activity in left and right M1 became more correlated during ipsilateral movements. We assume that, in the absence of rTMS, pcPM reduces the correlation by inhibiting adjacent M1. Evaluating the mechanistic details of inhibitory control is an important question for future studies involving alternative physiological methods as fMRI is largely insensitive to the dynamics of inhibitory systems (Waldvogel et al., 2000).

An alternative account for our results centers on the idea that the precentral premotor region may actively contribute 
to the control of the ipsilateral moving hand via uncrossed descending projections (Vulliemoz, Raineteau, \& Jabaudon, 2005). This hypothesis is supported by the observation that the spatial location of the left pcPM coincides with a region shown to elicit MEPs in ipsilateral hand muscles (Ziemann et al., 1999; Wassermann, Pascual-Leone, \& Hallett, 1994). According to this model, the change in M1 excitability we observed during ipsilateral actions simply reflects downstream information propagating to the adjacent premotor area as control demands increase. However, this model also fails to account for the correlation between left and right M1 and cannot explain why this correlation increases following rTMS to pcPM. Indeed, this account predicts that this correlation pattern should remain unchanged following rTMS or even decrease if coincidental signals from the upstream pcPM are attenuated.

The current results also provide a network level account for the presence of mirror movements in neurologically healthy populations (Cincotta \& Ziemann, 2008; Mayston et al., 1999). During unimanual actions, the desired motor command is mirrored in motor cortex, ipsilateral to the moving hand (see also Verstynen et al., 2007), perhaps as a way of providing additional computational resources while coordinating complex actions (Davare, Duque, Vandermeeren, Thonnard, \& Olivier, 2007). As movement complexity increases, this activation increases the possibility of incidental mirror movements. To compensate, neurons in the caudal region of dorsal premotor cortex are recruited to suppress this coupling between the motor cortices, effectively preventing the expression of unwanted mirror movements. Understanding the physiological mechanisms mediating the recruitment of ipsilateral motor regions and the interaction between these areas is a goal for future work.

\section{Acknowledgments}

We thank Mark D'Esposito, Steve Lehman, Winston Byblow, and Cathy Stinear for helpful discussions and Lisa Stewart for assistance with data collection. This project was funded by NIH grants NS30256 and NS40813.

Reprint requests should be sent to Timothy Verstynen, Learning Research and Development Center, University of Pittsburgh, Pittsburgh, PA 15213, or via e-mail: timothyv@pitt.edu.

\section{Notes}

1. The degrees of freedom differ for the three epochs because TMS was only applied during the movement phase for eight participants.

2. Examination of the simple correlation profile in this muscle shows a significant negative correlation between activity of the moving hand and MEPs in the stationary hand at approximately $200 \mathrm{msec}$ before movement onset. The mean intertap interval for this muscle was $272 \mathrm{msec}$. Thus, the correlation may be related to the fast oscillation rate of this muscle during simple movements. In contrast, the contraction rate in the ADM during complex actions is much longer, and a negative correlation would not be expected within the analysis window.

\section{REFERENCES}

Allen, E. A., Pasley, B. N., Duong, T., \& Freeman, R. D. (2007). Transcranial magnetic stimulation elicits coupled neural and hemodynamic consequences. Science, 317, 1918-1921.

Baumer, T., Dammann, E., Bock, F., Kloppel, S., Siebner, H. R., \& Munchau, A. (2007). Laterality of interhemispheric inhibition depends on handedness. Experimental Brain Research, 180, 195-203.

Beck, T. W., Housh, T. J., Johnson, G. O., Cramer, J. T., Weir, J. P., Coburn, J. W., et al. (2007). Does the frequency content of the surface mechanomyographic signal reflect motor unit firing rates? A brief review. Journal of Electromyography and Kinesiology, 17, 1-13.

Bestmann, S., Baudewig, J., Siebner, H., Rothwell, J., \& Frahm, J. (2003). Subthreshold high-frequency TMS of human primary motor cortex modulates interconnected frontal motor areas as detected by interleaved fMRI-TMS. Neuroimage, 20, 1685-1696.

Biswal, B., Yetkin, F., Haughton, V., \& Hyde, J. S. (1995). Functional connectivity in the motor cortex of resting human brain using echo-planar MRI. Magnetic Resonance in Medicine, 34, 537-541.

Brinkman, J., \& Kuypers, H. G. (1973). Cerebral control of contralateral and ipsilateral arm, hand and finger movements in the split brain rhesus monkey. Brain, 96, 653-674.

Cincotta, M., \& Ziemann, U. (2008). Neurophysiology of unimanual motor control and mirror movements. Clinical Neurophysiology, 119, 744-762.

Cisek, P., Crammond, D. J., \& Kalaska, J. F. (2003). Neural activity in primary motor and dorsal premotor cortex in reaching tasks with the contralateral versus ipsilateral arm. Journal of Neurophysiology, 89, 922-942.

Cramer, S. C., Finklestein, S. P., Schaechter, J. D., Bush, G., \& Rosen, B. R. (1999). Activation of distinct motor cortex regions during ipsilateral and contralateral finger movements. Journal of Neurophysiology, 81, 383-387.

Davare, M., Duque, J., Vandermeeren, Y., Thonnard, J. L., \& Olivier, E. (2007). Role of the ipsilateral primary motor cortex in controlling the timing of hand muscle recruitment. Cerebral Cortex, 17, 353-362.

Devanne, H., Lavoie, B., \& Capaday, C. (1997). Input-output properties and gain changes in the human corticospinal pathway. Experimental Brain Research, 114, 329-338.

Donchin, O., Gribova, A., Steinberg, O., Bergman, H., \& Vaadia, E. (1998). Primary motor cortex is involved in bimanual coordination. Nature, 395, 274-278.

Duque, J., \& Ivry, R. B. (2009). Role of corticospinal suppression during motor preparation. Cerebral Cortex, 19, 2013-2024.

Duque, J., Lew, D., Mazzocchio, R., Olivier, E., \& Ivry, R. B. (2010). Evidence for two concurrent inhibitory mechanisms during response preparation. Journal of Neuroscience, 30, 3793-3802.

Duque, J., Murase, N., Celnik, P., Hummel, F., Harris-Love, M., Mazzocchio, R., et al. (2007). Intermanual differences in movement-related interhemispheric inhibition. Journal of Cognitive Neuroscience, 19, 204-213.

Esselle, K., \& Stuchly, M. (1992). Neural stimulation with magnetic fields: Analysis of induced electric fields. IEEE Transactions on Biomedical Engineering, 39, 693-700.

Ferbert, A., Priori, A., Rothwell, J. C., Day, B. L., Colebatch, J. G., \& Marsden, C. D. (1992). Interhemispheric inhibition of the human motor cortex. Journal of Physiology, 453, 525-546.

Geyer, S., Matelli, M., Luppino, G., \& Zilles, K. (2000). Functional neuroanatomy of the primate isocortical motor system. Anatomy and Embryology (Berlin), 202, 443-474. 
Gilio, F., Rizzo, V., Siebner, H., \& Rothwell, J. (2003). Effects of right motor hand-area excitability produced by low-frequency rTMS over human contralateral homologous cortex. Journal of Physiology, 551, 563-573.

Hanakawa, T., Parikh, S., Bruno, M. K., \& Hallett, M. (2005). Finger and face representations in the ipsilateral precentral motor areas in humans. Journal of Neurophysiology, 93, 2950-2958.

Hayashi, M. J., Saito, D. N., Aramaki, Y., Asai, T., Fujibayashi, Y., \& Sadato, N. (2008). Hemispheric asymmetry of frequency-dependent suppression in the ipsilateral primary motor cortex during finger movement: A functional magnetic resonance imaging study. Cerebral Cortex, 18, 2932-2940.

Henderson, A. (2005). The bootstrap: A technique for data-driven statistics. Using computer-intensive analyses to explore experimental data. Clinica Chimica Acta: International Journal of Clinical Chemistry, 359, 1-26.

Ingram, J. N., Körding, K. P., Howard, I. S., \& Wolpert, D. M. (2008). The statistics of natural hand movements. Experimental Brain Research, 188, 223-236.

Johnson-Frey, S. H., Newman-Norlund, R., \& Grafton, S. T. (2005). A distributed left hemisphere network active during planning of everyday tool use skills. Cerebral Cortex, 15, 681-695.

Kawashima, R., Inoue, C. A., Sato, K., \& Fukuda, H. (1997). Functional asymmetry of cortical motor control in left-handed subjects. NeuroReport, 8, 1729-1732.

Kiebel, S. J., \& Holmes, A. (2004). The general linear model. In R. S. J. Frackowiak, K. J. Friston, C. D. Frith, R. J. Dolan, C. J. Price, S. Zeki, et al. (Eds.), Human brain function (pp. 749-779). San Diego, CA: Elsevier.

Kim, S., Ashe, J., Hendrich, K., Ellermann, J., Merkle, H., Ugurbil, K., et al. (1993). Functional magnetic resonance imaging of motor cortex: Hemispheric asymmetry and handedness. Science, 261, 616-617.

Kobayashi, M., Hutchinson, S., \& Schlaug, G. (2003). Ipsilateral motor cortex activation on functional magnetic resonance imaging during unilateral hand movements is related to interhemispheric interactions. Neuroimage, 20, 2259-2270.

Landau, S. M., Schumacher, E. H., Garavan, H., Druzgal, T. J., \& D'Esposito, M. (2004). A functional MRI study of the influence of practice on component processes of working memory. Neuroimage, 22, 211-221.

Mayston, M., Harrison, L., \& Stephens, J. (1999). A neurophysiological study of mirror movements in adults and children. Annals of Neurology, 45, 583-594.

Muellbacher, W., Facchini, S., Boroojerdi, B., \& Hallett, M. (2000). Changes in motor cortex excitability during ipsilateral hand muscle activation in humans. Clinical Neurophysiology, 111, 344-349.

Nagarajan, S. S., Durand, D., \& Hsuing-Hsu, K. (1997). Mapping location of excitation during magnetic stimulation: Effects of coil position. Annals of Biomedical Engineering, 25, 112-125.

Netz, J. (1999). Asymmetry in transcallosal inhibition. Electroencephalography and Clinical Neurophysiology Supplement, 51, 137-144.

Netz, J., Ziemann, U., \& Homberg, V. (1995). Hemispheric asymmetry of transcallosal inhibition in man. Experimental Brain Research, 104, 527-533.
Pasley, B. N., Allen, E. A., \& Freeman, R. D. (2009). State-dependent variability of neuronal responses to transcranial magnetic stimulation of the visual cortex. Neuron, 62, 291-303.

Rao, S., Binder, J., Bandettini, P. A., Hammeke, T., Yetkin, F. Z., Jesmanowicz, A., et al. (1993). Functional magnetic resonance imaging of complex human movements. Neurology, 43, 2311-2318.

Santello, M., Flanders, M., \& Soechting, J. F. (1998). Postural hand synergies for tool use. Journal of Neuroscience, 18, 10105-10115.

Santello, M., \& Soechting, J. F. (2000). Force synergies for multifingered grasping. Experimental Brain Research, 133, 457-467.

Shibasaki, H., Sadato, N., Lyshkow, H., Yonekura, Y., Honda, M., Nagamine, T., et al. (1993). Both primary motor cortex and supplementary motor area play an important role in complex finger movement. Brain, 116, 1387-1398.

Singh, L. N., Higano, S., Takahashi, S., Kurihara, N., Furuta, S., Tamura, H., et al. (1998). Comparison of ipsilateral activation between right and left handers: A functional MR imaging study. NeuroReport, 9, 1861-1866.

Stedman, A., Davey, N., \& Ellaway, P. (1998). Facilitation of human first dorsal interosseous muscle responses to transcranial magnetic stimulation during voluntary contraction of the contralateral homonymous muscle. Muscle \& Nerve, 21, 1033-1039.

Stinear, C. M., Walker, K. S., \& Byblow, W. D. (2001). Symmetric facilitation between motor cortices during contraction of ipsilateral hand muscles. Experimental Brain Research, 139, 101-105.

Tanji, J., Kazuhiko, O., \& Kazuko, C. S. (1988). Neuronal activity in cortical motor areas related to ipsilateral, contralateral, and bilateral digit movements of the monkey. Journal of Neurophysiology, 60, 325-343.

Todorov, E. (2004). Optimality principles in sensorimotor control. Nature Neuroscience, 7, 907-915.

Verstynen, T., Diedrichsen, J., Albert, N., Aparicio, P., \& Ivry, R. B. (2005). Ipsilateral motor cortex activity during unimanual hand movements relates to task complexity. Journal of Neurophysiology, 93, 1209-1222.

Verstynen, T., Spencer, R., Stinear, C. M., Konkle, T., Diedrichsen, J., Byblow, W. D., et al. (2007). Ipsilateral corticospinal projections do not predict congenital mirror movements: A case report. Neuropsychologia, 45, 844-852.

Vulliemoz, S., Raineteau, O., \& Jabaudon, D. (2005). Reaching beyond the midline: Why are human brains cross wired? Lancet Neurology, 4, 87-99.

Waldvogel, D., Gelderen, P. V., Muellbacher, W., Ziemann, U., Immisch, I., \& Hallett, M. (2000). The relative metabolic demand of inhibition and excitation. Nature, 406, 995-998.

Wassermann, E. M., Pascual-Leone, A., \& Hallett, M. (1994) Cortical motor representation of the ipsilateral hand and arm. Experimental Brain Research, 100, 121-132.

Weiss, E. J., \& Flanders, M. (2004). Muscular and postural synergies of the human hand. Journal of Neurophysiology, $92,523-535$.

Ziemann, U., Ishii, K., Borgheresi, A., Yaseen, Z., Battaglia, F., Hallett, M., et al. (1999). Dissociation of the pathways mediating ipsilateral and contralateral motor-evoked potentials in human hand and arm muscles. Journal of Physiology, 518, 895-906. 